\title{
Impact of correcting peak flow for nonlinear errors on air pollutant effect estimates from a panel study
}

\author{
D.J. Ward*, M.R. MIller**, S. Walters***, R.M. Harrison***, J.G. Ayres*
}

\begin{abstract}
Impact of correcting peak flow for nonlinear errors on air pollutant effect estimates from a panel study. D.J. Ward, M.R. Miller, S. Walters, R.M. Harrison, J.G. Ayres. (C) ERS Journals Ltd 2000.

ABSTRACT: Air pollutant effects are commonly investigated using panel studies employing daily measurement of changes in peak expiratory flow (PEF). Variable orifice PEF meters are inaccurate with a nonlinear relationship to actual PEF. The impact on a panel study of correcting these errors was examined.

Twice-daily PEF readings were taken by 147 9-yr old children for 8 weeks and corrected using an equation derived from the response of 32 Vitalograph meters to a servomechanism-controlled pump. Pollutant effect estimates for corrected and uncorrected readings were derived using a regression approach incorporating appropriate confounders.

Correction impacted little on mean PEF values (333.1-334.2 L. $\mathrm{min}^{-1}$ ), but did alter effect sizes. Nonsignificant nitrogen dioxide estimates for the entire panel decreased by up to $73 \%$, but, for symptomatic/atopic children, a significant 5-day mean result was lost (decrease in effect size from $\mathbf{- 2 . 5 3}$ to $-\mathbf{0 . 9 0 \%}$ per 10 parts per billion (ppb)) and lag 0 became significant (decrease from $-\mathbf{0 . 5 1}$ to $-1.22 \%$ per $10 \mathrm{ppb}$ ). Mass concentration estimates of particles with a 50\% cut-off aerodynamic diameter of $2.5 \mu \mathrm{m}$ moved in both directions $\left(-0.22\right.$ changed to $0.11 \%$ per $10 \mu \mathrm{g} \cdot \mathrm{m}^{-1}$ lag 3 and -0.29 to $-0.73 \%$ per $10 \mu \mathrm{g} \cdot \mathrm{m}^{-3}$ for the 5-day mean).

Correction of nonlinearity of peak expiratory flow meters influenced the overall outcome of this panel study, and the changes in effect estimates would be sufficient to alter the interpretation of some studies. For adults, a greater change in effect estimates may follow the larger correction required for their usual peak expiratory flow range. Eur Respir J 2000; 15: 137-140.
\end{abstract}

\begin{abstract}
*Heartlands Research Institute, Birmingam Heartlands Hospital, Bordesley Green East, Birmingham, B9 5SS, UK **Dept of Medicine, University of Birmingham, Selly Oak Hospital, Birmingham, UK ***Institute of Public and Environmental Health, University of Birmingham, Birmingham,
\end{abstract} UK

Correspondence: J.G. Ayres, Heartlands Research Institute, Birmingham Heartlands Hospital, Bordesley Green East, Birmingham, B9 5SS, UK. Fax. 441217724259

Keywords: Air pollution

childhood asthma

nonlinearity

panel study

peak flow

Received: February 21999

Accepted after revision September 151999

This study was funded by the Department of Health, UK.
The adverse health effects of air pollutants are now commonly accepted ranging from mortality and hospital admissions to respiratory symptoms and decrements in lung function. However, these effects, on a day-to-day basis, are small and so estimates of their magnitude may be substantially affected by inadequate control of confounding factors. In recent years, such estimates have been refined by the utilization of increasingly sophisticated study design and data analysis techniques $[1,2]$. One such study design for examining the relationship between short-term variations in pollutant concentrations and respiratory health is the panel study, in which individuals rather than populations are studied $[3,4]$. Preselected subjects make repeated, often daily, observations or measurements over periods of weeks to months that are related to pollutant levels measured over a similar time course. Subjects therefore, act as their own control, making this a powerful technique which removes the need to consider other factors which may impact on these end-points but do not themselves vary in a time-dependant way, such as atopy or age.

Daily changes in lung function and particularly peak expiratory flow (PEF) as a measure of airflow limitation are commonly used as an outcome in panel studies. In part, this relates to the availability of cheap durable hand-held meters, which are also widely used in clinical and occu- pational settings to assist in the diagnosis and homemonitoring of asthma. Such meters do not measure flow directly but rather incorporate a marker which moves in response to the force delivered by the airflow. The deflection of this marker is known to relate to maximal flow in a nonlinear way, the error profile varying according to meter design [5]. Standard meters that employ a linear scale will therefore produce readings which are not only inaccurate but also distort PEF variability across a range of flows. Such errors would be likely to alter the relationship between an exposure variable and a PEF record calculated using a "least squares" approach. Thus a pollutant effect estimate arrived at from multivariate linear regression analysis would not necessarily reflect the actual relationship between exposure and physiological or "true" PEF.

Meters can be calibrated and correcting equations derived by using a computerized pump system to deliver a very accurate flow $[5,6]$. Using such a system, it has been shown that treatment decisions based on a PEF management plan in severe asthmatics would have been significantly different for corrected versus uncorrected PEF records measured using a mini-Wright meter [7]. The opportunity was taken to examine and describe the effect on the final results that correcting PEF readings for nonlinearity would have on an air pollution panel study in children. 


\section{Materials and methods}

The air pollution panel study was carried out in an urban setting in the West Midlands, UK for two 8-week periods representing winter (January-March 1997) and summer (May-July 1997) conditions. Children aged 9 yrs in September 1996 recorded daily respiratory symptom scores and twice-daily PEF. As this analysis aimed to determine whether correction of nonlinear errors in PEF records had any effect on the results of a panel study rather than the size of any individual effect per se, data from a single monitoring period only were used. Summer afternoon PEF readings were chosen and, after 14 exclusions for inadequate data collection, records from 147 children ( $47 \%$ female) were available for analysis. From these data, pollutant effect estimates were calculated for both the PEF values recorded directly by the children and the PEF record after correction for PEF meter error.

\section{Panel study recruitment and monitoring}

All children from the appropriate year group in five primary schools were eligible. A parental questionnaire was used to identify those children with pre-existing symptoms and all children underwent spirometry and skin-prick testing to common aeroallergens. Parents reported wheezing in the previous year in 39/161 (24\%), which defined the "symptomatic" group, and 50/161 (31\%) yielded at least one positive skin test result, the "atopic" group. Nineteen (12\%) were both symptomatic and atopic. The panel were required to perform PEF measurement twice daily during the monitoring periods (at the start and end of the schoolday or equivalent, approximately between 08:30 and 09:00 h and between 15:15 and 15:45 h) and to record this information on a diary card collected weekly. The children and class teachers were instructed in the use of the PEF meter according to a standard protocol [8] and recorded only the highest of three blows on each occasion. Reasons for noncompletion of the PEF record were obtained from the children and confirmed by the teacher if school days were included.

The five schools were chosen on the basis of their close proximity to existing air pollution monitoring equipment (which was either part of the Enhanced Urban Network or provided by local councils) and major roads. The 24-h mean concentrations of nitrogen dioxide, total oxides of nitrogen, sulphur dioxide, ozone, carbon monoxide and the particulate measures mass concentration of particles with a $50 \%$ cut-off aerodynamic diameter of 10 (PM10) and 2.5 $\mu \mathrm{m}$ (PM2.5), were recorded along with measures of aerosol strong acidity and individual acid and anion species at two sites. The University of Birmingham Weather Service and the Pollen Research Unit, Worcester, UK, provided data on weather (daily maximum, mean and minimum temperature, mean relative humidity, barometric pressure, wind speed, total solar radiation and rainfall) and summer pollen counts respectively.

\section{Correction of peak expiratory flow results for meter error}

The children used the Vitalograph portable PEF meter of variable orifice type with a standard linear scale (Vitalograph, Buckingham, UK). A random sample of 32 (20\%) meters was selected from the winter and summer moni- toring periods for performance testing. In turn, each meter was subjected to a range of flows $\left(60-720 \mathrm{~L} \cdot \mathrm{min}^{-1}\right)$ from a computerized servomechanism-controlled pump system producing cusp-shaped flow profiles flows held at a peak for $12 \mathrm{~ms}$ [5], and the meter response (observed PEF) was read visually from the meter's own scale. Various mathematical models, including linear, quadratic and cubic functions, were fitted to the relationship between the mean response of all 32 meters and delivered flow (true PEF) and the best-fitting function determined (SPSS version 6.0 (SPSS Inc.), CURVEFIT command). This function acts as a correcting equation that can then be applied to all summer panel readings to produce a corrected dataset. A subset of four meters were tested an additional six times at each of four flows between 120 and $600 \mathrm{~L} \cdot \mathrm{min}^{-1}$ to assess the consistency of meter response.

\section{Panel study analysis}

Initial exploration of the summer PEF data was carried out using corrected data. Each individual child's data were first converted to daily z scores (deviation from individual mean PEF divided by the sd of the individual's summer afternoon PEF) and a mean $\mathrm{z}$ score was calculated for the entire panel on each day. This daily group value was used to explore the data for potential confounding variables because it reduces the impact of missing data from a highor low-reading child. Terms for time trend, days of the week, daily meteorological variables, pollen count and autocorrelation were considered for the final model and included on the basis of statistical significance $(\mathrm{p}<0.05)$ or visual impact on the residual plot. A similar process was conducted with the uncorrected PEF record to confirm that the same final model was appropriate.

The confounding terms identified were included as independent variables in single pollutant (lagged by up to 3 days or as the mean of lags $0-4$ ) regression analyses with the PEF record for each child (expressed as daily percentage deviation from mean PEF). Individual effect estimates were therefore obtained from the beta coefficients for each pollutant. The final results for the panel as a whole were obtained by pooling such individual estimates with each weighted by a factor derived from the inverse of its SEM to reduce the impact of children with a large SEM due to high variability or fewer data points. In this way, group effect estimates with $95 \%$ confidence intervals (CIs) for corrected and uncorrected PEF records were obtained for each pollutant at different lags.

\section{Results}

\section{Peak expiratory flow correction}

The results of performance testing the 32 meters are shown in figure 1 . On average, the meters overestimated at flows of up to $300 \mathrm{~L} \cdot \mathrm{min}^{-1}$ and then underestimated at higher flows with increasing errors for increasing flows. No deflection of the scale marker was seen for any of the meters at a flow of $60 \mathrm{~L} \cdot \mathrm{min}^{-1}$. The four meters tested for consistency of response showed no variation in reading off the scale visually. The mean meter response is shown in figure 2. From these values, the best fit correction equation was the quadratic, true flow $=0.0005$ measured $\mathrm{PEF}^{2}+0.7561$ measured $\mathrm{PEF}+23.3$ residual standard 


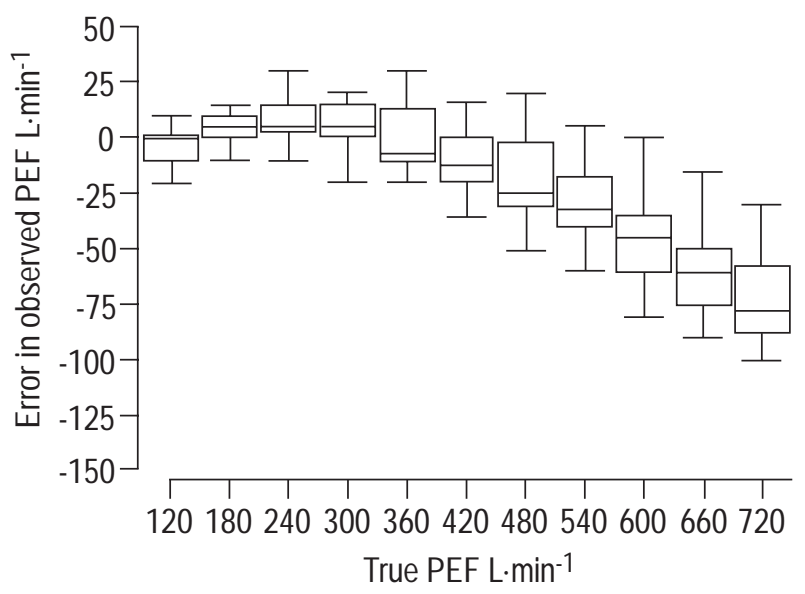

Fig. 1. - Error profile of 32 vitalograph peak flow meters at different flow rates delivered by a computerized servomechanism controlled pump. The box plots show the median and interquartile range, with the vertical bars representing the total range. PEF: peak expiratory flow.

deviation $\left(1.54 \mathrm{~L} \cdot \mathrm{min}^{-1}\right)$. The fit for this equation, which is very close, is also shown in figure 2 .

The mean PEF values fell into the range in which the size of the measured meter error was small and therefore correction had little effect on these values. The mean of all summer afternoon PEFs for the entire panel changed by only $1.1 \mathrm{~L} \cdot \mathrm{min}^{-1}(0.3 \%)$, from 333.1 to $334.2 \mathrm{~L} \cdot \mathrm{min}^{-1}$. However, a very slight increase in the overall range of values was seen. Values for the 25 th percentile, median and 75 th percentile averaged across all 147 subjects changed from $301.4,328.9$ and $356.5 \mathrm{~L} \cdot \mathrm{min}^{-1}$ respectively for uncorrected PEFs to 298.9, 328.6 and $359.4 \mathrm{~L} \cdot \mathrm{min}^{-1}$ after correction.

\section{Effects of peak expiratory flow correction on pollutant} effect estimates

PEF correction impacted on the final summer afternoon PEF results derived from pooled individual regression coefficients in a number of ways. Comparing results for effect estimates calculated from corrected and uncorrected PEF data demonstrated shifts in both the positive and

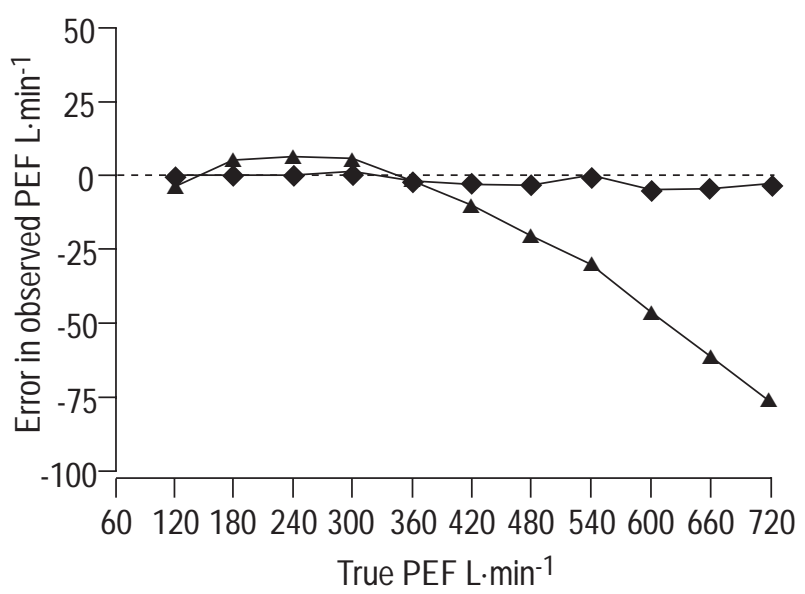

Fig. 2. - Mean error of 32 Vitalograph peak flow meters at different flow rates $(\boldsymbol{\Delta})$ and residual error after correction equation applied $(\boldsymbol{\bullet})$. PEF: peak expiratory flow.

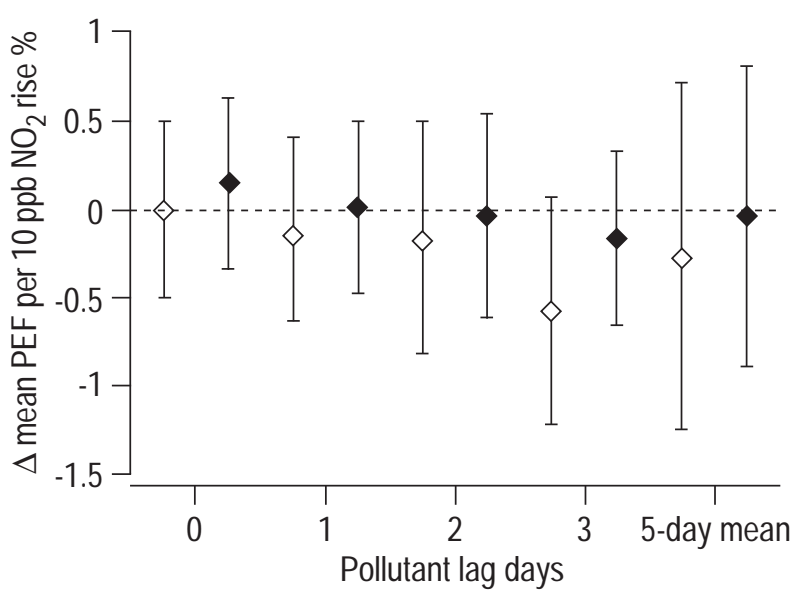

Fig. 3. - Effect estimates with $95 \%$ confidence intervals calculated for both uncorrected $(\diamond)$ and corrected peak expiratory flow (PEF) records $(\diamond)$. : change in; 5-day mean: lag $0-4$.

negative direction, with increases and decreases in absolute size and a widening and narrowing of the $95 \%$ CI. These outcomes are illustrated in figures 3-5, which show the results for $\mathrm{NO}_{2}$ and $\mathrm{PM} 2.5$ at all lags investigated. Figures 3 and 5 present the results for all panel members, whereas figure 4 includes only those who were both atopic and symptomatic, a group thought to be more sensitive to pollutant effects. The full results of the panel study, using corrected PEF readings only to determine the effect of pollution on lung function and symptoms, will be published elsewhere.

For the entire panel, $\mathrm{NO}_{2}$ effect estimates were all corrected in the positive direction with a narrowing of the $95 \%$ CI, and all but the same day result were decreased in absolute size by up to $73 \%$ (the effect estimate for $\mathrm{NO}_{2}$ lagged 3 days was corrected from -0.56 to $-0.15 \%$ per 10 parts per billion (ppb)). The apparent pattern of increasing effect with longer lag, approaching statistical significance for the 3-day lag result, is completely abolished by PEF correction. When only the "symptomatic/atopic" children were considered, the significant 5-day mean results were rendered nonsignificant with a decrease in effect size from

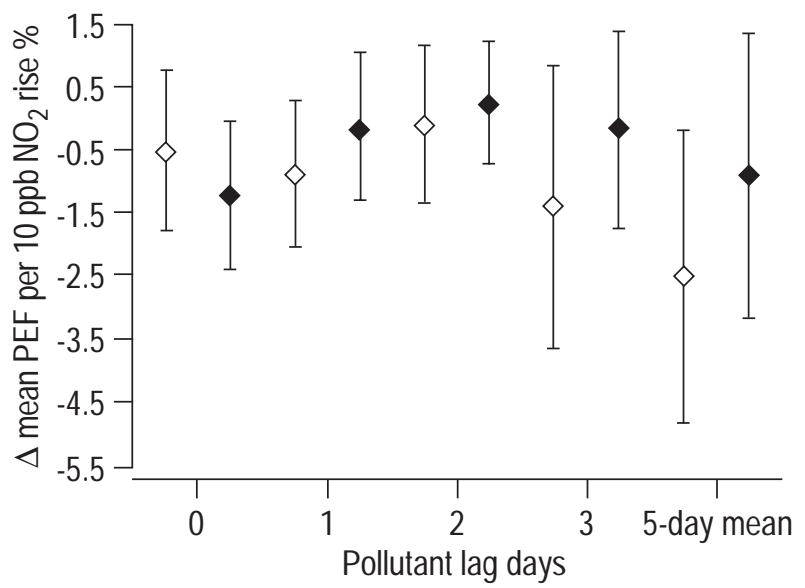

Fig. 4. - Effect estimates with $95 \%$ confidence intervals for subjects with both reported wheezing and a positive skin test only, calculated for both uncorrected $(\diamond)$ and corrected peak expiratory flow (PEF) records $(\diamond) . \triangle$ : change in; 5-day mean: lag $0-4$. 


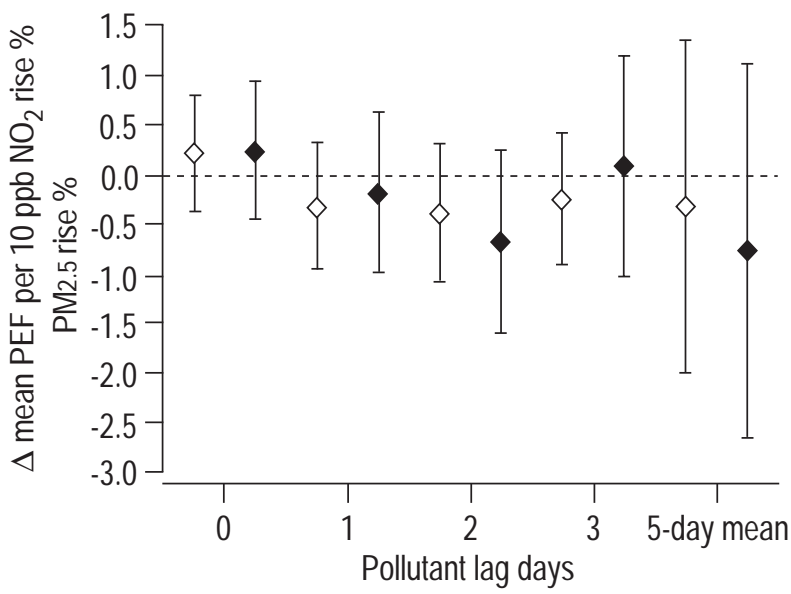

Fig. 5. - Effect estimates with 95\% confidence intervals calculated for both uncorrected $(\diamond)$ and corrected peak expiratory flow (PEF) records $(\diamond) . \triangle$ : change in; PM2.5: mass concentration of particles with a $50 \%$ cut-off aerodynamic diameter of $2.5 \mathrm{~mm}$; 5-day mean: lag $0-4$.

-2.53 to $-0.90 \%$ per $10 \mathrm{ppb}$ (64\% increase). In addition, correction produced a statistically significant result at lag 0 ; the effect estimate is incrased from -0.51 to $-1.22 \%$ per $10 \mathrm{ppb}$ (139\% increase). For the PM2.5, the results show a different pattern. PEF correction produced a widening of all the $95 \%$ CIs but changes to the effect estimates depended upon the lag considered. Shifts in both the positive and negative direction were seen, producing increases and decreases in absolute size of $>150 \%$ in the case of the 3 day lag (from -0.22 to $0.11 \%$ per $10 \mu \mathrm{g} \cdot \mathrm{m}^{-3}$ ) and 5-day mean (from -0.29 to $-0.73 \%$ per $10 \mu \mathrm{g} \cdot \mathrm{m}^{-3}$ ) results.

\section{Discussion}

In this panel study, the opportunity was taken to explore the effect of the recognized nonlinearity of PEF meters on health effect size coefficients. It was confirmed that PEF correction did affect the results of a panel study despite having little impact on the means and ranges of values seen. In absolute terms, the changes were small but, in relative terms, they led to important shifts in the direction and size of effect estimates and probable interpretation of results. Confidence intervals were also affected and could influence the acceptance of results which rely on the concept of statistical significance. For adults, PEF readings will be higher and the effects of nonlinearity using this particular meter will be greater. Similarly, the mean error of these Vitalograph meters was not as large as the error previously reported for the mini-Wright meter [7]. Correction in either of these circumstances may have a greater impact on detection of the presence or absence of a pollutant effect and the size of such estimates.

The effects of correction were not consistent across pollutants or lags and could not be easily predicted; there- fore, to determine the effect of correction on the results of similar studies, meter-specific corrections to the PEF record would need to be made and the data reanalysed. The size of effects on PEF reported from published panel studies are small $0.04-0.25 \%$ decrease in PEF per $10 \mu \mathrm{g} \cdot \mathrm{m}^{-3}$ rise in particulates) and are not consistently statistically significant between studies. A meta-analysis, however, suggested overall $0.08 \%$ decrement in PEF per $10 \mu \mathrm{g} \cdot \mathrm{m}^{-3}$ rise in PM10 [9]. Consequently, correction for nonlinearity could result in increases in effect size or removal of previously significant results.

To accurately quantify pollutant health effects based on a particular outcome requires an understanding of the physiology underlying that outcome and the performance of the equipment used to measure it. The authors suggest that existing adult panel data be reassessed using existing error equations for specific meters.

Acknowledgements. The authors would like to acknowledge the assistance of the Pollen Research Unit, Worcester, UK

\section{References}

1. Brunekreef B, Dockery DW, Krzyzanowski G. Epidemiologic studies on short-term effects of low levels of major ambient air pollution components. Environ Health Perspect 1995; 103 (Suppl. 2): 3-13.

2. Lebowitz MD. Epidemiological studies of the respiratory effects of air pollution. Eur Respir J 1996; 9: 1029-1054.

3. Brunekreef B, Hoek G, Roemer W, van der Zee S. Panel studies for investigating the acute health effects of air pollution. Eur Respir Rev 1998; 8: 53, 131-134.

4. Committee on the Medical Effects of Air Pollutants. Asthma and Outdoor Air Pollution. London, Her Majesty's Stationery Office, 1995.

5. Miller MR, Dickinson SA, Hitchings DJ. The accuracy of portable peak flow meters. Thorax 1992; 47: 904-909.

6. Pedersen OF, Rasmussen TR, Kjaergaard SK, Miller MR, Quanjer $\mathrm{PhH}$. Frequency response of variable orifice type peak flow meters: requirements and testing. Eur Respir $J$ 1995; 8: 849-855.

7. Miles JF, Tunnicliffe W, Cayton RM, Ayres JG, Miller MR. Potential effects of correction of inaccuracies of the mini-Wright peak expiratory flow meter on the use of an asthma self-management plan. Thorax 1996; 51: 403406.

8. Anonymous. Recommendations of the British Thoracic Society and the Association of Respiratory Technicians and Physiologists. Guidelines for the measurement of respiratory function. Respir Med 1994; 88: 165-194.

9. Dockery DW, Pope CA. Acute respiratory effects of particulate air pollution. Annu Rev Public Health 1994; 15: $107-132$. 